\title{
Erratum
}

\section{International Committee on Systematics of Prokaryotes Subcommittee on the taxonomy of phototrophic bacteria. Minutes of the meetings, 27 August 2003, Tokyo, Japan}

\author{
J. F. Imhoff and M. T. Madigan \\ International Journal of Systematic and Evolutionary Microbiology vol. 54, part 3, pp. 1001-1003 \\ On page 1002, in Table 1 of the printed and PDF versions of this article, the abbreviations \\ given for Rhodovulum and Rhodobaca are incorrect; they should be Rdv. and Rca., \\ respectively. Also, all abbreviations should end with a full stop. A new version of Table 1 , \\ which incorporates the aforementioned corrections, can be found below.
}

Table 1. Genera of anoxygenic phototrophic bacteria and recommended abbreviations

\begin{tabular}{|c|c|c|c|c|c|c|c|c|c|}
\hline $\begin{array}{l}\text { Chromatiaceae } \\
\gamma \text {-Proteobacteria }\end{array}$ & & \multicolumn{2}{|c|}{ Ectothiorhodospiraceae } & \multicolumn{2}{|c|}{ Purple non-sulfur bacteria } & \multicolumn{2}{|c|}{ Aerobic purple bacteria } & \multicolumn{2}{|l|}{ 'Green bacteria' } \\
\hline Chromatium & Chr. & Ectothiorhodospira & Ect. & Rhodospirillum & Rsp. & Acidiphilium & Acp. & Chlorobium & Chl. \\
\hline Amoebobacter & $A m b$. & Thiorhodospira & Trs. & Phaeospirillum & Phs. & Erythromicrobium & Erm. & Pelodictyon & Pld. \\
\hline Halochromatium & Hch. & & & Rhodobacter & $R b a$. & Erythromonas & Emn. & Ancalochloris & Anc. \\
\hline Isochromatium & Isc. & & & Rhodobium & Rbi. & Porphyrobacter & Por. & Chloroherpeton & Chp. \\
\hline Lamprocystis & $L p c$. & & & Rhodomicrobium & Rmi. & Roseococcus & Rsc. & 'Chloroflexaceae' & \\
\hline Marichromatium & Mch. & & & Rhodopila & Rpi. & Sandaracinobacter & San. & Chloroflexus & $C f l$. \\
\hline Rhabdochromatium & $R b c$ & & & Rhodoplanes & Rpl. & Roseivivax & Rsv. & Chloronema & $C \ln$ \\
\hline Thermochromatium & Tch. & & & Rhodopseudomonas & Rps. & Roseovarius & Rva. & Heliothrix & Htr. \\
\hline Thiocapsa & Tca. & & & Rhodospira & Rsa. & Roseibium & $R i b$. & Oscillochloris & Osc. \\
\hline Thiolamprovum & $T l p$ & & & Rhodoblastus & $R b l$. & Citromicrobium & Cmi. & Heliophilum & Hph. \\
\hline Thiopedia & Tpd. & & & Rhodobaca & Rca. & Methylobacterium & $M t b$ & Heliorestis & Hrs. \\
\hline Thiorhodococcus & $\operatorname{Trc}$ & & & Roseospirillum & Rss. & Acidisphaera & Acs. & & \\
\hline Thiorhodovibrio & $\operatorname{Tr} v$ & & & $\beta$-Proteobacteria & & Rubritepida & Rut. & & \\
\hline Thiospirillum & Tsp. & & & Rhodocyclus & $R c y$. & Staleya & Stl. & & \\
\hline Thioalkalicoccus & Tac. & & & Rhodoferax & $R f x$. & $\beta$-Proteobacteria & & & \\
\hline Thioflavicoccus & $T f \mathcal{c}$. & & & Rubrivivax & Rvi. & Roseateles & Rst. & & \\
\hline Thiobaca & Tba. & & & & & & & & \\
\hline
\end{tabular}

